\title{
Symmetry Of Irreducible Cluster Energy And The Theory Of Elastic Constant Crystals: Co as an Example
}

\author{
Gufan Yu.M. \\ Research Institute of Physics \\ Southern Federal University \\ Rostov-on-Don, Russia \\ yu.gufan@gmail.com \\ Kukin O.V. \\ Research Institute of Physics \\ Southern Federal University \\ Rostov-on-Don, Russia \\ yu.gufan@gmail.com
}

\author{
Naskalova O.V. \\ Faculty of Physics and ICT \\ Chechen State University \\ Grozny, Russia \\ naskalova_o@mail.ru \\ Fomin G.V. \\ Faculty of Physics \\ Southern Federal University \\ Rostov-on-Don, Russia \\ yu.gufan@gmail.com
}

\begin{abstract}
The paper proposes a new method of transferable calculation of elastic constants of the second, third and fourth orders. The method is based on the assumption that the potential energy of bulk crystals may be presented as the decomposition into irreducible energies of two, three, and four atomic clusters. To justify this decomposition, we proved that the irreducible energy of atomic clusters obeys additional symmetry $\mathrm{O}_{3} \times \mathrm{N}_{\mathrm{Cl}}$, which depends on the number of atoms constituting clusters $\left(\mathrm{N}_{\mathrm{Cl}}\right)$, and not depends on cluster structure. Additional symmetry allows defining the bases invariants, which depend on interatomic distances, and irreducible energies of clusters are depended on bases invariants only. In turn, these dependences made it possible to calculate the elastic modulus of complexes that exist in different crystal modifications as functions of the same phenomenological parameters of the model of interatomic interactions. Then we presented an example of the calculation of elastic constants BCC, HCP and FCC of the metal phases of $\mathrm{Co}$ polymorphs. The results confirm that the proposed method is transferable and makes it possible to calculate the elastic moduli of the second, third and fourth orders with the accuracy not worse than was achieved using the quantum chemistry models.
\end{abstract}

Keywords-elastic moduli, Cauchy ratio, irreducible representation

\section{INTRODUCTION}

The solution of the majority of problems of condensed matter physics requires the knowledge of interatomic interaction energy dependence $E_{P}\left(\left\{\vec{r}_{j}\right\}\right)$ on geometrical characteristics of interacting atoms. In general, this dependence is unknown even for ideal crystals consisting of similar atoms. Therefore, the type of dependence of interatomic interaction energy $E_{P}\left(\left\{\vec{r}_{j}\right\}\right)$ on vectors $\vec{r}_{j}$ defining the arrangement of atoms and phenomenological parameters $E_{P}\left(\left\{\vec{r}_{j}\right\}\right)$ is always postulated through the comparison of calculation results and experimentally defined crystal characteristics. A variety of literature sources refer to dozens of different types of semi-empirical potentials of interatomic interactions ( $E_{P}$ models) considering pair, triatomic and tetratomic interactions [1-10].

Despite ever-increasing popularity of various models (abinitio calculations) based on quantum chemistry methods, the interest in the search of semi-empirical models of interatomic interaction potentials is still relevant. Quantum and chemical calculations are based on numerical solution of the Schrödinger equation for the Hamiltonian of the polyatomic system, which is written within the mean field approximation. The type of the Hamiltonian of polyatomic systems is unknown and is postulated in the same manner. Literature sources consider dozens of options to implement the quantum chemistry methods (various options of pseudopotential, models based on density functional approximation $[4]$, etc.).

A key advantage of the models of potentials compared to quantum and chemical methods is their substantially smaller insistence on electronic computational resources. This allows solving a wider range of atomistic modeling problems. The calculation of atomic structures and properties of polyatomic objects with unknown structure presents the greatest interest. Such problems include the calculation of technologically important border properties of crystal phases, modeling of structure and properties of defects, as well as definition of structure and properties of small clusters. The solution of such problems requires the potential model with transferability properties. In other words, in terms of phenomenological (adjustable) parameters obtained through the comparison of calculation results within the model with known experimental data for one modification of a matter the model shall be able to forecast parameters and properties of other polymorphic modifications of this matter. 
The embedded atom model (EAM) became the most popular. For example, the work [5] suggests the EAM model, which, according to authors, may be used for atomistic modeling of $\mathrm{Co}$ microstructures within a wide range of temperatures. The authors' conclusion on the use of this potential is based on the fact that phenomenological parameters of a model are calculated according to known basic properties of FCC and HCP of Co phases. The model used in [5] contains 12 phenomenological parameters, which were determined via minimization of average quadratic deviation of parameters of a lattice, elastic modulus of the second order, vacancy energy and other HCP properties of the Co phase. Various options to adjust the parameters of the EAM model, which approximate the HCP properties of the Co phase with similar accuracy. The final choice of model parameters was made through the comparison of the FCC properties of the Co phase predicted by potential with experimental data. The numerical data of elastic modulus of the second order of HCP and FCC Co phases approximated for this model and their comparison with the experiment and the results obtained in this study are shown in Tables 2 and 4.

Despite the fact that within the model [5] it was possible with satisfactory degree of accuracy to approximate the set of experimental data for Co FCC and HCP, this model is not transferable and cannot be used in calculations of Co atomic structures existing in nature. For satisfactory approximation of experimental data, it was necessary to adjust the values of 12 model parameters with the accuracy of up to 7 significant digits. Similar accuracy is not coherent with the accuracy of experimental data, which define these parameters. Thus, for example, according to pilot study [6], the accuracy of the elasticity module s11 of the FCC Co phase makes $8.8 \%$. In other words, the model [5] is structurally unstable and hence, small changes of phenomenological parameters of the EAM model (within the $7^{\text {th }}$ digit after comma) lead to considerable changes of predicted physical properties of various Co modifications. Besides, it shall be noted that the given study does not include the values of elastic modulus of the third order predicted in EAM, which experimental values are known for HCP cobalt phase. This fact is also connected with the structural instability accepted in [5]. The work [7] demonstrates the calculation of embedded atom parameters for 12 cubic metals ( $\mathrm{Ag}, \mathrm{Al}, \mathrm{Au}, \mathrm{Cu}, \mathrm{Fe}, \mathrm{K}, \mathrm{Li}, \mathrm{Mo}, \mathrm{Na}, \mathrm{Nb}, \mathrm{Ni}$ and $\mathrm{Rb}$ ) approximating the values of elastic moduli of the second and third orders. For this purpose, the authors of [5] had to use the model with 21 phenomenological (adjustable) parameters and the required accuracy of their determination of up to 10 significant digits.

The purpose of this study was to demonstrate the possibility of a new approach based on the principles of symmetry, which is aimed to search the adequate transferable and structurally stable models of a potential allowing forecasting properties of several polymorphic modifications of crystals formed by the atom of one element. The paper considers BCC, FCC and $\mathrm{HCP}$ of the Co phase as an example.

\section{MODELS OF POTENTIALS TAKING INTO ACCOUNT IRREDUCIBLE ENERGY OF INTERACTION WITHIN PAIR, TRIATOMIC AND TETRATOMIC CLUSTERS}

Let us assume that the total potential energy of a crystal $\left(E_{P}\left\{\vec{r}_{i j}\right\}\right)$ may be presented as the infinite sum of irreducible potential energies $\left(\varepsilon_{N}\right)$ of interaction between $\mathrm{N}$ atoms of a cluster, consisting of all pairs $\left(\varepsilon_{2}\left(\vec{r}_{i j}\right)\right)$, triatomic $\left(\varepsilon_{3}\left(\vec{r}_{i j}, \vec{r}_{j k}, \vec{r}_{k i}\right)\right)$ and tetratomic $\left(\varepsilon_{4}\left(\vec{r}_{i j}, \vec{r}_{i l}, \vec{r}_{i k} \vec{r}_{j k}, \vec{r}_{j l}, \vec{r}_{k l}\right)\right)$ atoms located on crystal lattice sites. Here $\left\{\vec{r}_{i j}\right\}$ - vector at the origin of atom $i$ to the center of atom $j$. If some set of elements $\vec{a}_{i}$ is considered, then hereinafter the symbol $\left\{\vec{a}_{i}\right\}$ defines the entire set. If two clusters are partially blocked, i.e. some atoms belonging to one cluster also belongs to the second and include several coinciding atoms, then the direct unambiguous division of potential energies of these clusters is impossible.

Therefore, the first stage of the microscopic theory is to propose and develop a method of dependence of irreducible energies of atoms interaction in each cluster containing mentally held atom on a relative positioning of all atoms making a cluster. The method shall lead to such calculations, which will make it possible to take the next step in the creation of the microscopic (molecular) theory of macroscopic characteristics of crystals. The second stage is to find a method of summation of irreducible potential energies of all clusters so that the obtained sum equals the total potential energy of a crystal regardless of the fact that some clusters contain the same atoms.

The direct solution of both problems does not exist. Thus, for instance, the explicit form of dependence of irreducible potential energy of a cluster consisting of three atoms $\left(\varepsilon_{3}\right)$ on their relative position, i.e. on vectors $\vec{r}_{12}, \vec{r}_{23}, \vec{r}_{31}$ is unknown and cannot be defined. However, the additional symmetry of analytical dependence of irreducible energies on coordinates of atoms allows defining some typical features of this dependence $\varepsilon_{3}\left(\vec{r}_{12}, \vec{r}_{23}, \vec{r}_{31}\right)$. The analytical dependence $\varepsilon_{N}$ on vectors $\vec{r}_{i j}, \vec{r}_{l m}$ is defined by the group of symmetries $G_{N}$ of irreducible potential energy of the cluster containing $N$ particles: $\varepsilon_{N}\left(\vec{r}_{12}, \vec{r}_{23}, \ldots \vec{r}_{N-1, N}\right)$. The $G_{N}$ group is equal to the direct product of $3 \mathrm{D}$-space rotation group $O(3)$ by the permutation group $P_{N}$ of $N$ numbers of similar atoms within a cluster: $G_{N} \equiv O(3) \otimes P_{N}$.

Thus, for example, the energy of atom interactions in clusters consisting of two atoms is the distance function between centers of atoms in a cluster: $\varepsilon_{2}=\frac{1}{2} \sum_{i, j} \varepsilon_{2}\left(\vec{r}_{i j}\right)$. The consequence of this symmetry $\left(G_{2}\right)$ of irreducible energy of pair interactions includes the known Cauchy ratios, which for elastic moduli of the second order of cubic crystals may be presented as follows: $c_{12}=c_{44}$ (by Vogt: $1 \equiv x x, 2 \equiv y y, 3 \equiv z z$, $4 \equiv y z, 5 \equiv x z, 6 \equiv x y)$. It is known that the Cauchy ratios are not satisfied for any crystal of cubic symmetry in a wide interval of external changes. 
Then, let us consider a cluster consisting of three atoms $i$, $j$, $k$, which centers are connected by vectors $\vec{r}_{i j}, \vec{r}_{j k}$ and $\vec{r}_{k i}=-\vec{r}_{i j}-\vec{r}_{j k}$. For this cluster the influence of symmetry comes down to the fact that the irreducible energy of a cluster may always be presented as the function of three vector invariants of the $G_{3}$ group [8]:

$$
\begin{gathered}
I_{1}=\vec{r}_{i j}^{2}+\vec{r}_{j k}^{2}+\vec{r}_{k i}^{2}, I_{2}=\vec{r}_{i j}^{2} \vec{r}_{j k}^{2}+\vec{r}_{j k}^{2} \vec{r}_{k i}^{2}+\vec{r}_{k i}^{2} \vec{r}_{i j}^{2}, \\
I_{3}=\vec{r}_{i j}^{2} \vec{r}_{j k}^{2} \vec{r}_{k i}^{2} .
\end{gathered}
$$

As shown in [8], the distortion of the Cauchy ratios for elastic moduli of the second order and similar ratios between elastic moduli of the third and higher orders is the consequence of $G_{3}$ symmetry. However, the additional ratios of symmetry not being the consequence of internal and external symmetry of elastic moduli remain between elastic moduli of the third and higher orders. For cubic crystals these ratios are as follows: $c_{123}-3 c_{144}+2 c_{456}=0$ for elastic moduli of the third order and $c_{1123}+2 c_{1456}-c_{1144}-2 c_{1244}=0$ for elastic modulus of the fourth order.

The irreducible energy of the cluster consisting of four atoms may always be presented as the function of nine vector invariants $Y_{n}(n=1-9)$ of the $G_{4}$ group, which analytical form is given in [9]. Using the example of one invariant $Y_{n}$, the [8] shows that $G_{4}$ distorts all additional ratios not connected with accurate internal and external symmetry of elastic moduli.

Thus, the symmetry analysis made it possible to conclude that any potential model of interatomic interactions claiming for adequate description of elastic nonlinear anisotropic characteristics of crystals shall sufficiently consider the irreducible energies of interactions in clusters consisting of pair, triatomic and tetratomic atoms.

The method of calculation of invariants on various lattice sites was proposed and developed in [8]. The invariants characterizing the clusters from three atoms on FCC and BCC lattice sites as functions of a cubic elementary cell are given in [8] and [10] respectively. We also proposed methods to calculate the total potential energy of a crystal as a sum of cluster energies based on numerical values of invariants and not requiring the calculation of certain invariants of the basis $[8,10]$.

The given study suggests 27 potential models of interatomic interactions, each of which considers irreducible interactions within clusters consisting of two, three and four atoms.

In order for the energy of a crystal calculated within a model to present the additive function, the pair, triatomic and tetratomic interactions shall be decreased with a distance not more slowly than $1 /|\vec{r}|^{3}, 1 /|\vec{r}|^{6}$ and $1 /|\vec{r}|^{9}$ [10] respectively. Let us consider the models, which dependence of pair, triatomic and tetratomic interactions of attraction decreases in absolute magnitude depending on the distance between atoms. The repulsive potentials decrease with higher degrees depending on distance:

$$
\begin{aligned}
& \varepsilon_{m n}=-\sum_{i, j} \frac{a}{\vec{r}_{i j}^{6}}+\sum_{i, j} \frac{b}{\vec{r}_{i j}^{12}}-\sum_{i, j, k} \frac{q_{1}}{I_{m}^{8 / v_{m}}}+\sum_{i, j, k} \frac{q_{2}}{I_{m}^{14 / v_{m}}}- \\
& -\sum_{i, j, k, l} \frac{w_{1}}{Y_{n}^{12 / v_{n}}}+\sum_{i, j, k, l} \frac{w_{2}}{Y_{n}^{18 / v_{n}}}+
\end{aligned}
$$

In (2) one fixed invariant $I_{m}$ taken from (1) and $Y_{n}$ from [9] is used as characteristics of triatomic and tetratomic interactions; indexes $m=1 \ldots 3$ and $n=1 \ldots 9$ designate numbers of corresponding invariants forming the basis for $\left(G_{3}\right)$ and $\left(G_{4}\right)$ respectively; vm and vn - degrees of invariants number $m$ and $n$. Parameters $a, b, q_{1}, q_{2}, w_{1}$ and $w_{2}-$ phenomenological parameters defining the model of a particular matter.

The sums in (2) are calculated for cobalt BCC, FCC and HCP through the summation along sites of the corresponding crystal lattice A1, A2 and HCP.

\subsection{Model selection criteria}

1) It is known that the stability criteria of a stable state of a matter impose restrictions on potential phenomenological parameters of models intended to describe the given state. Thus, for example, one of stability conditions of the LennardJones potential model of pair interactions (special case of models (2) obtained at $q_{1}=q_{2}=w_{1}=w_{2}=0$ ) is the minimum of function $\varepsilon_{2}\left(\vec{r}_{i j}^{2}\right)$, i.e. $a, b>0$. The use of a similar criterion to a series of models used in (2) comes down to the requirement of the minimum of functions $\varepsilon_{m n}\left(\tau_{1}\right)$ at $\tau_{1}=\tau_{1}{ }^{\exp }$. This is also applicable for the minimum $\varepsilon\left(\tau_{2}\right)$ at $\tau_{1}=\tau_{2}{ }^{\exp }$. In case with Co these requirements are not satisfied for 18 out of 27 models: $\varepsilon_{12}, \varepsilon_{13}, \varepsilon_{14}, \varepsilon_{15}, \varepsilon_{16}, \varepsilon_{17}, \varepsilon_{18}, \varepsilon_{22}, \varepsilon_{23}, \varepsilon_{24}, \varepsilon_{25}, \varepsilon_{27}, \varepsilon_{28}$, $\varepsilon_{34}, \varepsilon_{35}, \varepsilon_{36}, \varepsilon_{37}$ and $\varepsilon_{39}$. These models cannot be further considered as adequately describing the A1 and A2 state of Co and are thus withdrawn from further analysis.

2) The Born's stability criteria [11] imposed on elastic moduli of the second order typical for a stable state of cubic crystals may be presented as follows: $c_{11}, c_{12}, c_{44}>0, c_{11}>c_{12}$, $c_{44}$. For models $\varepsilon_{19}$ and $\varepsilon_{29}$ the modulus $c_{44} \simeq 0$, which contradicts the third condition. Models $\varepsilon_{11}$ and $\varepsilon_{21}$ do not satisfy the last condition. Models $\varepsilon_{26}$ and $\varepsilon_{33}$ forecast nonphysical values of elastic moduli of the third order, namely $c_{112}, c_{112}>0$, which contradicts the criteria similar to the Born's stability criteria but for elastic moduli of the third order.

Further verification of remaining three models $\varepsilon_{31}, \varepsilon_{32}$ and $\varepsilon_{38}$ was based on the comparison of the forecast values of elastic moduli of the second order of cobalt A1 and A2 with values obtained experimentally in [6, 14-16]. It turned out that the values of elastic moduli $c_{44}$ for $\mathrm{A} 1$ and $\mathrm{A} 2$ of Co phases forecasted in model $\varepsilon_{31}$ coincide better with experimental values by $5-9 \%$. Despite the fact that such error in definition of the module $c_{44}$ does not go beyond the error of its experimental state, let us be limited to the results obtained within the verified model $\varepsilon_{31}$. 
III. CALCULATION OF PHENOMENOLOGICAL PARAMETERS OF A MODEL AND ELASTIC MODULI FOR CO MONOCRYSTALS

The calculation of elastic moduli requires the summation crystal lattice sides and thus result in various dependence of elastic moduli in different crystal modifications of a crystal on phenomenological parameters of microscopic model of interatomic interactions (2). For example, the dependence of the elastic modulus $c_{11}$ for crystals with FCC structure on phenomenological parameters of model $\varepsilon_{11}$ :

$$
\begin{aligned}
& c_{11}=\left(-0.04184 a+4.216 \cdot 10^{-4} b\right)+ \\
& +\left(-6.552 \cdot 10^{-4} q_{1}+1.058 \cdot 10^{-7} q_{2}\right)+ \\
& +\left(-6.815 \cdot 10^{-8} w_{1}+9.318 \cdot 10^{-13} w_{2}\right) .
\end{aligned}
$$

To calculate the phenomenological parameters of the theory $a, b, q_{1}, q_{2}, w_{1}$ and $w_{2}$ in each out of 27 models $\varepsilon_{m n}$ (3) only 6 experimentally established values were used: $\tau_{1}{ }^{\exp }=$ $1.775 \AA, c_{11}=2.25 \cdot 10^{2} \mathrm{hPa}, c_{12}=1.60 \cdot 10^{2} \mathrm{hPa}$ for A1 phase taken from [6] and $\tau_{2}{ }^{\text {exp }}=1.409 \AA, c_{11}=1.93 \cdot 10^{2} \mathrm{hPa}, c_{12}=$ $1.70 \cdot 10^{2} \mathrm{hPa}$ for A2 phase given in works $[12,13]$. It shall be noted that the BCC structure of Co is not evident in volume single-crystal samples, but is observed in the "extended" single-crystal epitaxial Co films with the thickness of $357 \AA$ [12] and $202 \AA$ [13], as well as under negative pressure when Co clusters are removed from Au matrix during cooling [13]. The comparison of values calculated within the models with similar experimental values leads to the system of six linear algebraic equations for each model $\varepsilon_{m n}$. Table 1 shows the calculated values of phenomenological parameters for model $\varepsilon_{31}$ to demonstrate the structural stability of the theory developed through the study. The values of phenomenological parameters are presented in units: $[a]=\left[\tau^{6}\right] \cdot 10^{2} \mathrm{hPa},[b]=$ $\left[\tau^{12}\right] \cdot 10^{2} \mathrm{hPa},\left[q_{1}\right]=\left[\tau^{8}\right] \cdot 10^{2} \mathrm{hPa},\left[q_{2}\right]=\left[\tau^{14}\right] \cdot 10^{2} \mathrm{hPa},\left[w_{1}\right]=$ $\left[\tau^{12}\right] \cdot 10^{2} \mathrm{hPa}$ and $\left[w_{2}\right]=\left[\tau^{18}\right] \cdot 10^{2} \mathrm{hPa}$.

TABLE I. VALUES OF PHENOMENOLOGICAL PARAMETERS OF A MODEL (2) CALCULATED FOR COBALT MONOCRYSTALS

\begin{tabular}{|c|c|c|c|c|c|}
\hline $\boldsymbol{a}$ & $\boldsymbol{b}$ & $\boldsymbol{q}_{\mathbf{1}}$ & $\boldsymbol{q}_{\mathbf{2}}$ & $\boldsymbol{w}_{\mathbf{1}}$ & $\boldsymbol{w}_{\mathbf{2}}$ \\
\hline 20.7 & 4590 & 249 & $7.31 \cdot 10^{4}$ & $-1.41 \cdot 10^{7}$ & $-4.15 \cdot 10^{11}$ \\
\hline
\end{tabular}

Table 1 shows that the accuracy of all six phenomenological parameters does not exceed three significant digits, which is coherent with the accuracy of experimental values used for their calculation. This fact distinguishes the proposed models (2) from EAM models [5, 7] widely used in literature and a number of other popular models, which are partially presented in [1].

\section{RESULTS AND CONCLUSIONS}

Table 2 shows the calculation of elastic moduli of the second and third orders of Co crystals with A1 structure obtained using the verified model $\varepsilon_{31}$. The elastic moduli are written as $10^{2} \mathrm{hPa}$. The lines $2-4$ of Table 2 show the experimental values of elastic moduli of the second order of films obtained in $[6,15,16]$. The fifth line shows experimental values of elastic moduli of the second and third orders for Co$32 \% \mathrm{Ni}$ solid solution. The sixth or seventh lines show data regarding Ni single-crystals. The lines 8 and 9 show the results of calculations within the DFT model [17] and the EAM model [5]. The comparison of results made it possible to note that in $[5,17]$ the elastic moduli of the third order are not calculated since it requires the introduction of a bigger number of phenomenological parameters of these models, which makes the procedure of accurate (with the accuracy of up to 10 digits) adjustment of parameters complicated for satisfactory agreement of values of elastic moduli of the second and third order with the experiment.

TABLE II. VALUES OF ELASTIC MODULI OF THE SECOND AND THIRD ORDERS OF COBALT CRYSTALS OF WITH A1 STRUCTURE

\begin{tabular}{|c|c|c|c|c|c|c|c|c|c|}
\hline & $c_{11}$ & $c_{12}$ & $c_{44}$ & $c_{111}$ & $c_{112}$ & $c_{165}$ & $c_{123}$ & $c_{144}$ & $c_{456}$ \\
\hline Exp [6] & 2.25 & 1.6 & 0.92 & - & - & - & - & - & - \\
\hline Exp [15] & 2.23 & 1.86 & 1.1 & - & - & - & - & - & - \\
\hline Exp [16] & 2.38 & 1.58 & 1.28 & - & - & - & - & - & - \\
\hline $\begin{array}{l}\text { Exp [22] } C_{0}- \\
32^{\circ} \mathrm{Ni}\end{array}$ & 2.387 & 1.553 & 1.315 & -25.47 & -12.12 & -10.22 & -0.14 & -1.65 & -1.23 \\
\hline $\begin{array}{c}\text { Exp [19, 20] } \\
\mathrm{Ni}\end{array}$ & 2.612 & 1.508 & 1.317 & -25.25 & -15.59 & -9.08 & 0.05 & -2.66 & -1.63 \\
\hline Exp[21]Ni & - & - & - & -23.7 & -14.75 & -8.67 & 0.46 & -2.15 & -0.44 \\
\hline DFT [17] & 3.25 & 1.89 & 1.56 & - & - & - & - & - & - \\
\hline EAM[5] & 2.757 & 1.589 & 1.082 & - & - & - & - & - & - \\
\hline $\boldsymbol{E}_{31}$ & $\mathbf{2 . 2 5}$ & $\mathbf{1 . 6}$ & $\mathbf{0 . 8 4 4}$ & $\mathbf{- 1 8 . 8}$ & $-\mathbf{1 0 . 8}$ & $-\mathbf{7 . 8 6}$ & $\mathbf{- 3 . 0 5}$ & $\mathbf{- 0 . 7 2}$ & $\mathbf{0 . 3 0 5}$ \\
\hline
\end{tabular}

Poor measurement accuracy of elastic moduli of the third order shall also be considered in the comparison of calculation results of elastic moduli of the third order with the experiment. According to [18], the maximum measurement accuracy of elastic moduli makes about $10 \%, c_{112}-15 \%, c_{166}-50 \%, c_{123}$ $-100 \%, c_{144}-300 \%$ and $c_{456}-100 \%$. The comparison of elastic moduli of the third order forecasted in model $\varepsilon_{31}$ shows good agreement with the data obtained for $\mathrm{Co}-32 \% \mathrm{Ni}$ and Ni solution.

Table 3 shows the calculated values of Co elastic moduli A2 (elastic moduli are written as $10^{2} \mathrm{hPA}$ ). The experimental values of elastic moduli of the second order presented in [14] are given in the second line. The third line shows values calculated through the DFT model [17].

TABLE III. VALUES OF ELASTIC MODULI OF THE SECOND AND THIRD ORDERS OF COBALT CRYSTALS OF WITH A2 STRUCTURE

\begin{tabular}{|c|c|c|c|c|c|c|c|c|c|}
\hline & $c_{11}$ & $c_{12}$ & $c_{44}$ & $c_{111}$ & $c_{112}$ & $c_{166}$ & $c_{123}$ & $c_{144}$ & $c_{456}$ \\
\hline $\operatorname{Exp}[22]$ & 1.93 & 1.7 & 0.48 & - & - & - & - & - & - \\
\hline DFT [17] & 1.93 & 2.41 & 1.31 & - & - & - & - & - & - \\
\hline$\varepsilon_{31}$ & $\mathbf{1 . 9 3}$ & $\mathbf{1 . 7}$ & $\mathbf{1 . 0 4}$ & $\mathbf{- 1 6 . 1}$ & $\mathbf{- 8 . 9 3}$ & $-\mathbf{6 . 3 5}$ & $\mathbf{- 8 . 4 5}$ & $-\mathbf{6 . 8 3}$ & $\mathbf{- 6 . 1 0}$ \\
\hline
\end{tabular}

Table 4 shows parameters of a lattice and elastic moduli of the second order of the HCP Co phase forecasted in model $\varepsilon_{31}$ taking into account the phenomenological parameters $a, b, q_{1}$, $q_{2}, w_{1}$ and $w_{2}$ calculated according to parameters of a lattice and elastic moduli of the second order within A1 and A2 structures (elastic moduli are written as $10^{2} \mathrm{hPA}$ ).

TABLE IV. PARAMETERS OF A LATTICE AND ELASTIC MODULI OF THE SECOND ORDER OF THE HCP CO PHASE

\begin{tabular}{|c|c|c|c|c|c|c|}
\hline & $\tau_{\text {rny }}$ & $c_{11}$ & $c_{33}$ & $c_{12}$ & $c_{13}$ & $c_{44}$ \\
\hline Exp [23] & 2.51 & 3.071 & 3.581 & 1.650 & 1.027 & 0.755 \\
\hline Error [24] & & $2-4 \%$ & $2-4 \%$ & $4-7 \%$ & $15 \%$ & $2-4 \%$ \\
\hline IXS [24] & & 2.93 & 3.39 & 1.43 & 0.90 & 0.78 \\
\hline US [24] & & 2.95 & 3.35 & 1.59 & 1.11 & 0.71 \\
\hline GGA [24] & & 3.31 & 3.69 & 1.61 & 2.43 & 0.94 \\
\hline LDA [24] & & 2.97 & 3.42 & 1.31 & 0.82 & 0.96 \\
\hline EAM [5] & 2.52 & 3.119 & 3.594 & 1.469 & 1.196 & 0.917 \\
\hline$\varepsilon_{31}$ & $\mathbf{2 . 5}$ & $\mathbf{3 . 0 7}$ & $\mathbf{3 . 6 8 6}$ & $\mathbf{1 . 5 3}$ & $\mathbf{1 . 5 1}$ & $\mathbf{0 . 7 7 2}$ \\
\hline
\end{tabular}


The experimental values of elastic moduli of the second order presented in [23] are given in the first line of Table 4. The lines 2-5 show experimental values of elastic moduli calculated in various DFT models [24] different from each other by the choice of models of pseudo-potential, electronic correlations and exchange interactions. The line 6 contains data obtained through the EAM model [5]. The values calculated in model $\varepsilon_{31}$ are given in line 7. It is clear that the model $\varepsilon_{31}$ better forecasts values of elastic moduli of the second order of the HCP cobalt phase compared to other numerical calculations found in literature.

The values of elastic moduli of the third order of the HCP cobalt phase forecasted in model $\varepsilon_{31}$ are given in Table 5. The line 2 of Table 4 shows the experimental values of elastic moduli of the third order of the HCP cobalt phase presented in [25]. The line 3 - calculation results given in [26] and the line 4 - values calculated in model $\varepsilon_{31}$.

TABLE V. VALUES OF ELASTIC MODULI OF THE THIRD ORDER OF COBALT CRYSTALS WITH HCP STRUCTURE

\begin{tabular}{|c|c|c|c|c|c|c|c|c|c|c|}
\hline & $c_{111}$ & $c_{222}$ & $c_{333}$ & $c_{112}$ & $c_{113}$ & $c_{133}$ & $c_{123}$ & $c_{144}$ & $c_{155}$ & $c_{344}$ \\
\hline $\begin{array}{c}\text { Exp } \\
{[25]}\end{array}$ & -67.1 & -57.88 & -63.47 & -14.54 & -7.66 & -5.11 & -4.29 & 1.33 & -14.86 & -2.1 \\
\hline $\begin{array}{l}\text { Teor } \\
{[26]}\end{array}$ & -40.97 & -50.01 & -42.81 & -14.32 & -2.24 & -10.7 & -3.11 & -2.68 & -2.68 & -10.7 \\
\hline $\boldsymbol{\varepsilon}_{31}$ & $-\mathbf{3 7 . 4}$ & $-\mathbf{3 1 . 7}$ & $-\mathbf{3 6 . 5}$ & $-\mathbf{6 . 6 5}$ & $-\mathbf{6 . 6 0}$ & $-\mathbf{1 2 . 0}$ & $-\mathbf{3 . 0 3}$ & $-\mathbf{1 . 4 9}$ & $-\mathbf{3 . 3 0}$ & $-\mathbf{8 . 3 8}$ \\
\hline
\end{tabular}

Values $c_{111}, c_{222}$ and $c_{333}$ obtained in works $[25,26]$ through the method of successive approximations differ markedly from values obtained by the approximation of the dependence of deformation tensor components on stress components.

\section{Acknowledgements}

Yu.M. Gufan is grateful to the Research Institute of Physics of the Southern Federal University for financial support (internal grant No. BCH0110-11/2017-42 (within a state task 3.6386.2017/BCH).

\section{References}

[1] S. Erkoc, Empirical many-body potential energy functions used in computer simulations of condensed matter properties, Phys. Rep., vol. 278(2). pp. 79-105, 1997.

[2] S. Erkoc, Empirical Potential Energy Functions Used in Simulations of Materials Properties, in Annual Reviews of Computational Physics, vol. 9, D. Stauffer, Ed. 2001, pp.332.

[3] D. Y. Li, X. F. Wu, T. The effect of stress on soft modes for the phase transformation in a Ti-Ni alloy I. The stress-induced transformation and soft modes, Philosophical Magazine A, vol. 63 (3), pp. 585-601, 1991.
[4] P. Hohenberg, W. Kohn, Inhomogeneous Electron Gas, Phys. Rev. B., vol. 136(3), pp. B864-871, 1964.

[5] G. P. Purja Pun, Y. Mishin, Embedded-atom potential for hep and fcc cobalt, Phys. Rev. B., vol. 86, pp. 134116, 2012.

[6] J. Gump, X. Hua, M. Chirita, R. Sooryakumar, M.A. Tomaz, G.R. Harp, Elastic constants of face-centered-cubic cobalt, J. Appl. Phys., vol. 86(11), pp. 6005-6009, 1999.

[7] S. Chantasiriwan, F. Milstein, Embedded-atom models of 12 cubic metals incorporating second- and third-order elastic-moduli data, Phys. Rev. B., vol. 58(10), pp. 5996-6005, 1998.

[8] A.Yu. Gufan, O.V. Kukin, Yu.M. Gufan, A.Yu. Smolin, Model of three-particle interactions and the theory of nonlinear deformation of crystals, Fiz. Tverd. Tela, vol. 54( 4), pp. 770-781, 2012.

[9] I.A. Osipenko, O.V. Kukin, A.Yu. Gufan, Yu.M. Gufan, Polyatomic interactions in the theory of moduli of elasticity of a high order: General theory, Fiz. Tverd. Tela, vol. T. 55(12), pp. 2289-2296, 2013.

[10] Yu.M. Gufan, O.V. Kukin, A.Yu. Smolin, A new method for calculating three-particle interaction in the theory of modulus elasticity, Bulletin of the Russian Academy of Sciences: Physics, vol. 75, pp. 1267-1273, 2011.

[11] M. Born, K. Huang, Dynamical Theory of Crystal Lattices. Oxford: Oxford University Press, 1962, pp. 462.

[12] G.A. Prinz, Stabilization of bec Co via Epitaxial Growth on GaAs, Phys. Rev. Lett., vol. 54(10), pp. 1051-1054, 1985.

[13] S. Subramanian, R. Sooryakumar, G.A. Prinz, B.T. Jonker, Y.U. Idzerda, Elastic constants of body-centered-cubic cobalt films, Phys. Rev. B, vol. 49(24), pp. 17319-17324, 1994.

[14] R. Bauer, E. Bischoff, E.J. Mittemeijer, Natural formation of bec Co: Initial stage of $\mathrm{Co}$ precipitation in supersaturated $\mathrm{Au}_{90} \mathrm{Co}_{10}$, Phys. Rev. B, vol. 81, pp. 094113, 2010.

[15] B. I. Min, T. Oguchi, and A. J. Freeman, Structural, electronic, and magnetic properties of Co: Evidence for magnetism-stabilizing structure, Phys. Rev. B, vol. 33, pp. 7852, 1996.

[16] B.W. Bennett, G.W. Shannette, Acoust. Lett., vol. 4, pp. 99, 1980.

[17] G.Y. Guo, H.H. Wang, Gradient-Corrected Density Functional Calculation of Elastic Constants of Fe, Co and $\mathrm{Ni}$ in bcc, fcc and hep Structures, Chin. J. Phys., vol. 38(5), pp. 949-961, 2000.

[18] 18. B.E. Powell, M.J. Skove, Measurement of Higher-Order Elastic Constants, Using Finite Deformations, Phys. Rev., vol. 174(3), pp. 977983, 1968.

[19] Ch. Kittel, Introduction to solid state physics. Wiley, 1996, pp. 689.

[20] V.P. Sarma, P.J. Reddy, Third-Order Elastic Constants of Single Crystal of Ni at 80K, Phys. Stat. Sol. (a), vol. 16, pp. 413-418, 1973.

[21] A.S. Johal, D.J. Dunstan, Reappraisal of experimental values of thirdorder elastic constants of some cubic semiconductors and metals, Phys. Rev. B, vol. 73, pp. 024106, 2006.

[22] W.F. Weston, A.V. Granato, Cubic and hexagonal single-crystal elastic constants of a cobalt-nickel alloy, Phys. Rev. B., vol. 12, pp. 5355-5362, 1975.

[23] H.J. McSkimin, Measurement of the Elastic Constants of Single Crystal Cobalt, J. of Appl. Phys., vol. 26(4), pp. 406-409, 1955.

[24] D. Antonangeli, M. Krisch, G. Figuet, D.L. Farber, C.M. Aracne, J. Badro, F. Occelli, H. Requardt, Elasticity of Cobalt at High Pressure Studied by Inelastic X-Ray Scattering, Phys. Rev. Lett., vol. 93(21), pp. 215505, 2004

[25] Y.K. Yogurtcu, G.A. Saunders, Phil. Mag., vol. 52(6), pp. 833-846, 1985.

[26] R. Ramji Rao, A. Ramanand, Thermal expansion and bulk modulus of cobalt, J. Low. Temp., vol. 26, pp. 365-377, 1977. 